\title{
Processo de trabalho de auxiliares e técnicos de enfermagem na Atenção Básica à Saúde
}

\author{
Work process of nurses' aides and nursing technicians in Primary Health Care
}

Proceso de trabajo de auxiliares y técnicos de enfermería en la Atención Primaria de Salud

\author{
Tauani Zampieri Cardoso', Maria José Bistafa Pereira", Lucas Vinco de Oliveira Campos'"', \\ Silvia Matumoto", Silvana Martins Mishima", Cinira Magali Fortuna", Osmar de Oliveira Cardoso ${ }^{\text {IV }}$ \\ ' Universidade de São Paulo, Escola de Enfermagem de Ribeirão Preto, \\ Programa de Enfermagem em Saúde Pública. Ribeirão Preto-SP, Brasil. \\ "Universidade de São Paulo, Escola de Enfermagem de Ribeirão Preto, Departamento de Enfermagem Materno-Infantil \\ e Saúde Pública, Programa de Enfermagem em Saúde Pública. Ribeirão Preto-SP, Brasil. \\ I"' Prefeitura Municipal de Sertãozinho, Secretaria de Administração. Sertãozinho-SP. Universidade de São Paulo, \\ Escola de Enfermagem de Ribeirão Preto, Programa de Enfermagem em Saúde Pública (Mestrando). Ribeirão Preto-SP, Brasil. \\ Iv Universidade Federal do Piauí, Departamento de Bioquímica e Farmacologia, \\ Núcleo de Estudos em Saúde Pública. Teresina-Pl, Brasil.
}

Submissão: 13-09-2010 Aprovação: 08-01-2012

\section{RESUMO}

Esta investigação teve como objeto a prática de técnicos e auxiliares de enfermagem em um serviço de Atenção Básica à Saúde do município de Ribeirão Preto-SP. Os objetivos foram: caracterizar o processo de trabalho desses agentes; analisar suas concepções sobre os elementos constitutivos do processo de trabalho e identificar e analisar as dificuldades e facilidades no desenvolvimento do processo de trabalho, na perspectiva da integralidade. O estudo foi descritivo e qualitativo. Para a coleta de dados, utilizaramse a observação participante e a entrevista semiestruturada e os dados foram tratados com análise temática. Constatou-se o predomínio da organização do processo de trabalho para atender pessoas com necessidades de enfrentar doenças/problemas de saúde e encontraram-se potencialidades para o desenvolvimento do processo de trabalho na integralidade da atenção. Sugerimos a prática da educação permanente, a qualificação da escuta e novas políticas de formação desses trabalhadores.

Descritores: Atenção Primária à Saúde. Trabalho. Auxiliares de Enfermagem. Equipe de Enfermagem.

\begin{abstract}
This research had as its object the practice of nurses' aides and nursing technicians in service of Primary Health Care of the city of Ribeirão Preto-SP, Brazil. The aims were: characterize the work process of these agents, analyze his concepts on the constituent elements of working process and identify and analyze the difficulties and facilities in developing the work process from the perspective of integrality. It was descriptive and qualitative. To collect the data were used participant observation and the semi-structured interview and the data were dealt with thematic analysis. It was found the predominance of the organization of the work process to assist people with the need to deal with diseases/health problems and met potential for development of the work process for integrality health care. It is suggested the practice of permanent education, the qualification to listening and new training policies for these workers.
\end{abstract}

Key words: Primary Health care; Work; Nurses' aides; Nursing, staff.

\section{RESUMEN}

Esta investigación tuve por objeto la práctica de técnicos y auxiliares de enfermería al servicio de Atención Primaria de Salud del municipio de Ribeirão Preto-SP, Brasil. Los objetivos fueron: caracterizar el proceso de trabajo de estos agentes, analizar sus conceptos sobre los elementos constitutivos del proceso de trabajo y identificar y analizar las dificultades y facilidades en el desarrollo del proceso de trabajo desde la perspectiva de la integridad. La encuesta fue descriptivo y cualitativo. Para la recopilación de datos fue utilizada la observación participante y la entrevista semiestructurada y los datos fueram tratados con análisis temático. Se encontró un predominio de la organización del proceso de trabajo para satisfacer las personas con necesidades para hacer frente a enfermedades/ problemas de salud, y se reunió el potencial para el desarrollo del proceso de trabajo para la atención integral de salud. Se sugiere la práctica del aprendizaje permanente, la calificación de escuchar y nuevas políticas de formación para estos trabajadores.

Palabras clave: Atención Primaria de Salud. Trabajo. Auxiliares de Enfermería. Grupo de Enfermería. 


\section{INTRODUÇÃO}

O Movimento da Reforma Sanitária Brasileira resultou na aprovação na Constituição Federal da saúde como direito de todos e dever do Estado. Com isso, instituiu-se, em 1988, o Sistema Único de Saúde (SUS) regido pelos princípios da universalidade, integralidade e equidade da atenção, descentralização da gestão dos serviços, regionalização e hierarquização das unidades prestadoras de atendimento e participação da população no controle social ${ }^{(1,2)}$.

Para assegurar os direitos constitucionais em meio à complexidade conjuntural, avançar na consolidação do SUS e materializar suas diretrizes, mudanças foram necessárias, sendo que estas foram decisivas para a implantação de práticas da Atenção Básica à Saúde (ABS), nas políticas de saúde do Estado brasileiro. Com isso, ocorreu a incorporação de diversas categorias profissionais, bem como a ampliação de outras, na rede de assistência à saúde, incluindo os trabalhadores de enfermagem de nível médio.

Desde então, diversas estratégias vêm sendo adotadas, e muitos progressos já foram alcançados nas instâncias políticas, assistenciais e educacionais para a construção de uma prática de atenção à saúde voltada para a integralidade. No entanto, ainda existem muitos desafios a serem enfrentados, inclusive no que se refere à formação e ao processo de trabaIho dos profissionais auxiliares de enfermagem (AEs) e técnicos de enfermagem (TEs) na ABS.

Os AEs e TEs constituem um contingente significativo de trabalhadores nos serviços de saúde, e, na enfermagem, os AEs representam a maior força de trabalho ${ }^{(3)}$. No entanto, pouco se investiga como a prática desses trabalhadores tem se processado frente às perspectivas de mudanças no modelo de produção em saúde.

Essa situação provocou o desenvolvimento deste estudo, pois a prática desses trabalhadores pode se constituir em uma estratégia para facilitar as transformações indispensáveis para obtermos um sistema de saúde voltado para as necessidades da população(4).

Neste estudo consideramos a enfermagem não apenas como uma profissão com competência técnico-científica, mas como uma prática social, como trabalho, pois ela estabelece relações com as dimensões econômicas, culturais e sociais de uma sociedade e com outros trabalhos, participando da conformação das práticas de saúde e sendo conformada de acordo com o contexto em que se insere, sofrendo transformações no decorrer da história(5,6).

Como quadro teórico deste estudo, recorremos à fundamentação do processo de trabalho, com ênfase no processo de trabalho em saúde ${ }^{(5-7)}$, a fim de buscarmos compreender o processo de produção das ações em saúde.

Trabalho humano são atividades que transformam algo que havia antes em algo que corresponde à satisfação de uma necessidade, sendo esta transformação intencional, ou seja, o homem concebe e planeja o que vai produzir ${ }^{(7)}$.

Ao conceber e planejar as ações em saúde, os trabalhadores partem do que identificam como necessidade de saúde que deverá ser satisfeita, e a produção dessas ações engloba os elementos constitutivos do processo de trabalho: objeto, instrumentos, produto e finalidade que, por sua vez, encontram-se articulados e estão sustentados em uma concepção de homem e do processo saúde-doença.

O objeto de trabalho é a matéria-prima do trabalho, algo reconhecido como passível de transformações para se obter o produto. Ao reconhecer neste algo potencialidades para atender a uma dada necessidade, os trabalhadores operam com instrumentos, recursos para a transformação e obtenção do produto, e ao operar tais instrumentos transformam também a compreensão sobre seu trabalho, assim como as subjetividades produzidas, e seus desejos conformam os modos de agir desses trabalhadores.

O reconhecimento das necessidades de saúde e sua íntima relação com a finalidade do trabalho e com os demais elementos constitutivos do processo de trabalho quando operam são atravessados pela concepção de homem e de processo saúde-doença e, também, são conformados por processos históricos e sociais que determinam a organização das práticas de saúde.

Nessa perspectiva, apreender o desenvolvimento do processo de trabalho dos AEs e TEs na ABS permitiu-nos perceber como o princípio da integralidade tem entremeado as práticas desses trabalhadores.

Neste estudo, optamos por abordar o princípio da integralidade da atenção por considerarmos que todos os princípios do SUS têm interface e apresentam interdependência e complementaridade, de tal modo que o comprometimento de qualquer um dos demais princípios interfere diretamente no da integralidade ${ }^{(2)}$. A opção também se respalda por ser este princípio o que menos avançou na implementação do SUS, sendo um desafio a ser enfrentado.

Tomaremos a integralidade referindo-nos ao ser humano e ao sistema de saúde, reconhecendo que cada qual se constitui numa totalidade, não compartimentalizando as ações de promoção, proteção e recuperação da saúde, sendo que as unidades constitutivas do sistema também se configuram em um todo indivisível, capaz de prestar assistência integral(8).

Foram objetivos deste estudo: caracterizar o processo de trabalho dos AEs e TEs de uma Unidade Básica de Saúde (UBS) do município de Ribeirão Preto-SP, na perspectiva da integralidade da atenção; analisar a concepção dos AEs e TEs sobre os elementos constitutivos do processo de trabalho na ABS; e identificar e analisar os aspectos dificultadores e facilitadores para desenvolver o processo de trabalho, na perspectiva da integralidade.

\section{PERCURSO METODOLÓGICO}

Estudo de natureza descritiva e abordagem qualitativa, realizado em uma UBS do município de Ribeirão Preto-SP, para o qual foram convidados a participar todos os trabalhadores de nível médio de enfermagem que atuavam nesta UBS, 11 AEs e 3 TEs. Compuseram a amostra 12 trabalhadores que concordaram em participar do estudo, sendo 9 AEs e 3 TEs.

Para a coleta de dados, optamos pela observação participante e a entrevista semiestruturada sendo utilizados, nas duas técnicas, roteiros com a finalidade de nortear e qualificar os dados obtidos. 
As observações participantes foram realizadas durante as jornadas de trabalho dos AEs e TEs na UBS, sendo que a pesquisadora permaneceu aproximadamente 2 horas e 30 minutos em cada setor ou atividade de trabalho, nos diferentes períodos de funcionamento da UBS (manhã, tarde e vespertino), totalizando aproximadamente 38 horas de observações que ocorreram no período de 17 de agosto a 2 de setembro de 2009 .

Os setores e atividades observados em que os AEs e TEs atuam neste serviço de saúde foram as salas de vacina, curativo, medicação, pré-consulta e pós-consulta, visita domiciliar e a coleta de materiais para exames laboratoriais. Os trabaIhadores também atuam no atendimento de ginecologia e obstetrícia, no entanto, a observação desta atividade não foi permitida devido à preocupação do médico com a privacidade das usuárias.

As entrevistas semiestruturadas foram gravadas e, posteriormente, transcritas, tiveram duração média de 1 hora e foram realizadas no período de 7 de janeiro a 23 de fevereiro de 2010. Para a análise dos dados obtidos, utilizou-se a análise temática(9).

As questões éticas desta pesquisa estão baseadas na Resolução 196/1996, sendo que este estudo obteve aprovação do Comitê de Ética em Pesquisa da Escola de Enfermagem de Ribeirão Preto da Universidade de São Paulo em 6 de maio de 2008, sob o Protocolo $n^{\circ}$ 0880/2008. Aos profissionais que concordaram em participar do estudo foi oferecido o Termo de Consentimento Livre e Esclarecido.

\section{ANÁLISE E DISCUSSÃO DOS DADOS}

A análise temática permitiu identificar duas distintas composições no processo de trabalho dos AEs e TEs. De modo predominante, encontramos a organização do processo de trabalho para atender pessoas com necessidade de enfrentar doenças/ problemas de saúde. Entretanto, a conformação das unidades temáticas encontradas revelou potencialidades para o desenvolvimento do processo de trabalho na direção da integralidade da atenção, como podemos visualizar no Quadro 1.

É importante sinalizar que optamos por dividir os elementos constitutivos do processo de trabalho para a análise e discussão dos dados para facilitar a compreensão, porém, como já discutido anteriormente, esses elementos estão intimamente articulados e constituem um mesmo processo de trabalho.

\section{Necessidades de saúde}

De modo predominante encontramos, nos depoimentos dos AEs e TEs, que as necessidades de saúde são interpretadas como sendo pessoas com necessidade de enfrentar doenças, conforme mostra o depoimento a seguir: A necessidade são as doenças, né? Enfrentá-las, seria isso, né? (...) No caso meu, ali, da vacina, a prevenção, né? Das doenças (A01).

As necessidades são detectadas a partir daquilo que é explicitado verbalmente pelos usuários, sem buscar identificar necessidades implícitas, o que não favorece a integralidade da atenção, na medida em que não abre brechas para que se revelem necessidades que vão além das queixas relacionadas às doenças, aos problemas de saúde ou aos sintomas trazidos pelos usuários. Com isso a organização do trabalho e as ações em saúde ficam pautadas na recuperação de corpos doentes.

Refletir e discutir sobre produção de saúde, a partir das necessidades de saúde, não é inerente ao cotidiano e tampouco fundamenta o processo de trabalho no dia a dia desses trabalhadores, em decorrência do modo fragmentado como este se organiza. Entretanto, uma prática orientada pelas necessidades de saúde poderia subsidiar ações que melhor correspondessem às necessidades de saúde da população pela qual esta UBS é responsável.

Os AEs e TEs também identificaram como necessidade de saúde pessoas que precisam conversar, relatando que os usuários procuravam o serviço de saúde e buscavam pela equipe de enfermagem com essa finalidade. No entanto, ainda que acoIhessem esta demanda, os próprios trabalhadores não consideravam essa conversa como parte do trabalho na saúde. A organização do trabalho não favorece esse acolhimento dos usuários, inclusive por não dispor de espaço físico que proporcione privacidade no encontro do trabalhador de saúde com o usuário.

Atender os usuários que carregam suas histórias de vida, conflitos, angústias e sofrimentos, problemas e dificuldades faz com que os trabalhadores de nível médio de enfermagem concebam também como necessidade dessas pessoas que procuram o serviço de saúde desabafar, chorar, conversar, como mostra o depoimento a seguir.

...tem umas pessoas que vêm na unidade, que às vezes elas precisam de uma conversa, duma força, elas chegam, tá passando por alguma dificuldade em casa, alguma coisa dentro de casa, aí, tudo que elas tão sentindo, aí, elas ficam

\begin{tabular}{|l|l|}
\hline Elementos constitutivos do processo de trabalho & \multicolumn{1}{c|}{ Unidades Temáticas } \\
\hline Necessidades de saúde & Pessoas com necessidade de enfrentar doenças; pessoas com necessidade de conversar. \\
\hline Objeto de trabalho em saúde & $\begin{array}{l}\text { Pessoas que procuram atendimentos; o doente com foco na queixa; recursos técnicos; } \\
\text { produtos. }\end{array}$ \\
\hline Recursos/instrumentos de trabalho em saúde & Tecnologias duras; tecnologias leve-duras; tecnologias leves. \\
\hline O produto do trabalho em saúde & Bem-estar físico; conforto mental; procedimentos técnicos. \\
\hline Finalidade do trabalho em saúde & $\begin{array}{l}\text { Recuperação do corpo doente; assistir quem não pode pagar; reduzir/evitar gastos; gar- } \\
\text { antir o direito à saúde. }\end{array}$ \\
\hline
\end{tabular}

Quadro 1 - Elementos constitutivos do processo de trabalho e respectivas unidades temáticas encontradas na análise dos dados 
desesperadas, aí atrapalha no serviço, elas faltam do serviço, né? (...) Muitas vezes, ela nem tá precisando daqui, né? Tá precisando de a gente dar um tempo, conversar (A06).

Ao identificar a conversa como necessidade de saúde, esses trabalhadores sinalizam potencialidade para uma atenção à saúde na perspectiva da integralidade, na medida em que abrem espaço para identificar e buscar recursos e estratégias que atendam às necessidades que não se encontram no âmbito biológico.

\section{Objeto de trabalho em saúde}

O objeto de trabalho em saúde foi predominantemente reconhecido pelos AEs e TEs como a doença ou o doente.

Seria, a doença, o doente, é mais ou menos isso, né? (...) Aqui na unidade de saúde, geralmente é o doente (A11).

Tomar como objeto de trabalho o doente, como pode ser observado, com foco em sua queixa, mais uma vez desenha uma prática conformada em ações que visam a recuperar o corpo e fragmentam o homem, focalizando seus aspectos biológicos e a doença. Encontramos, também, o estar doente como condição para procurar o serviço de saúde. Desse modo, as ações dos AEs e TEs se tornam auxiliares do trabalho médico, a quem cabe o diagnóstico, a prescrição do tratamento ou encaminhamentos.

Houve o reconhecimento de recursos técnicos como objeto de trabalho: verificar a pressão, repassar conhecimento, medicação, paciência, seringa, agulha, entre outros. Esse reconhecimento pode ser fruto da intensa fragmentação da assistência que afasta os trabalhadores da possibilidade de enxergar o ser humano que atendem. A fragmentação da assistência e a redução das ações dos AEs e TEs a subsidiárias do trabalho médico resultam da divisão técnica e social do trabalho que desapropria os trabalhadores de nível médio de enfermagem dos resultados do processo de trabalho.

Os AEs e TEs reconheceram, também, o bem-estar e a assistência como objeto de trabalho e outros se mostraram incertos sobre o que seria o objeto de trabalho na ABS. Os embaraços e as incertezas podem propiciar a abertura de discussões e reflexões acerca do objeto de trabalho em saúde.

Potencialidades para uma prática de atenção à saúde mais ampliada e quiçá na perspectiva da integralidade podem ser antevistas quando os trabalhadores identificam as pessoas que procuram atendimento na UBS como objeto de trabalho, conforme o excerto abaixo, pois há possibilidades de considerarem o homem em sua complexidade como sujeito social.

Olha, importante é a pessoa que vem aqui procurar a gente, é a mais importante, porque se não vier não tem como, o que a gente vai, vai trabalhar, vai ganhar, né? Mas eu acho assim, o cliente é o principal papel, o papel principal, que vem e procura (A06).

Assim, estamos diante do limite dessa leitura que se restringe às pessoas que buscam atendimento na UBS, reforçando uma atenção à saúde individual e pontual em que planejamentos e ações de cunho coletivo são restritos e limitados, sendo que quase não foram observados neste estudo. Um ponto a ser considerado é a grande população da área de abrangência atendida pela UBS, sendo que os trabalhadores conhecem os usuários que procuram atendimento na UBS e aqueles para os quais realizam visitas domiciliares. Entretanto, dificilmente podemos dizer que os trabalhadores conhecem a população pela qual são responsáveis.

\section{Recursos/instrumentos de trabalho em saúde}

Os recursos/instrumentos do processo de trabalho em saúde serão abordados de acordo com a (re)conceituação de Merhy ${ }^{(10)}$ que classifica as tecnologias em saúde como duras, leve-duras e leves. As tecnologias duras englobam instrumentais complexos, equipamentos para tratamentos, exames e a organização de informações, as tecnologias leve-duras abrangem os saberes profissionais estruturados como a clínica, a epidemiologia e os saberes de outros profissionais que compõem a equipe de saúde, enquanto as tecnologias leves são produzidas no trabalho vivo, em ato, em um processo de relações que resultam do encontro entre trabalhadores e usuários dos serviços de saúde.

Dentro das tecnologias duras, os trabalhadores relataram ser necessário ter materiais para realizar curativos, atendimentos ginecológicos e obstétricos, medicação, aferir a pressão arterial e temperatura, glicosimetria, demais exames realizados pelos usuários e equipamentos de proteção individual.

Os AEs e TEs mencionaram, ainda, a necessidade de ter disponível eletrocardiograma e balança eletrônica, o que mostra preocupação e desejo de melhorar a qualidade da assistência prestada na UBS para se alcançar maior resolubilidade e evitar encaminhamentos. No entanto, não foram observadas discussões em equipe acerca dos recursos/instrumentos necessários ou reflexões a respeito de conceitos, normas, políticas e diretrizes da ABS.

Um fator apontado pelos AEs e TEs como gerador de sofrimentos, medos e conflitos é o acolhimento dos usuários que procuram a UBS sem agendamento prévio e apresentam queixas ou problemas, pois esses usuários acabam conformando uma demanda que não será atendida. As decisões relacionadas aos encaminhamentos, como dispensar o usuário sem atendimento médico, orientá-los a retornar em outro horário ou dia à UBS ou procurar outro serviço de saúde, são comunicadas pelos AEs e TEs.

Nesse momento do processo de trabalho há dificuldades para realizar ações na perspectiva da integralidade, na medida em que esses trabalhadores sentem-se despreparados para atuar, pois essa situação em que se conforma uma demanda que não será atendida, não foi abordada em seus cursos de formação. Isso sugere um distanciamento da formação dos AEs e TEs com a realidade dos serviços de $A B S$, na medida em que a formação está predominantemente voltada para o trabalho nos hospitais que exige e dispõe de um conjunto de tecnologias diferentes da ABS.

Há ainda a recusa do médico em realizar atendimentos além do estipulado, e a demanda trazida pelos AEs e TEs como prioridade não é acolhida por esse trabalhador. 
Nesta unidade de saúde parece não haver discussões sobre a regulamentação do exercício da enfermagem no que diz respeito à competência dos AEs e TEs para atuar no acolhimento dos usuários que procuram o serviço de saúde sem agendamentos prévios com queixas ou problemas de saúde.

Muitos recursos e estratégias têm sido adotados para fortalecer a ABS como a Política de Humanização que incentiva e qualifica a prática do acolhimento nos serviços de saúde ${ }^{(11)}$ e, neste sentido, a elaboração e adoção de fluxogramas, a classificação de riscos e vulnerabilidade, assim como a escuta ampliada das necessidades dos usuários podem ser estratégias muito efetivas, pois habilitam os trabalhadores a identificar as necessidades dos usuários e a buscar recursos na própria UBS, em outros níveis de atenção ou ainda em equipamentos sociais de outros setores ou na própria comunidade para a resolução desses problemas.

Observamos, ainda, o envolvimento dos trabalhadores da UBS em situações apresentadas pelos usuários como dificuldades financeiras, por exemplo. No entanto, os recursos utilizados foram de natureza assistencialista e caritativa, com a disponibilização de recursos próprios diante de demandas sociais.

Outra questão geradora de conflitos e inseguranças no processo de trabalho é o atendimento de urgências e emergências. Quando o usuário apresenta sua queixa ou problema, esses trabalhadores têm dificuldade em identificar se é uma situação de urgência ou emergência. Sabemos que a formação é insuficiente e que é essencial para a ABS que os trabalhadores sejam qualificados, se quisermos imprimir efetividade em suas ações ${ }^{(12)}$.

Dentro das tecnologias leve-duras, os trabalhadores AEs e TEs reconheceram a necessidade de ter formação para trabaIhar na saúde bem como de conhecimentos técnico-científicos. Apontaram, ainda, a necessidade de realizar cursos e atualizações. Consideramos importante sinalizar que, ao falarem da formação e de cursos, referem-se ao conhecimento relacionado ao corpo, às doenças e aos procedimentos, como no fragmento seguinte, o que converge com os demais elementos constitutivos do processo de trabalho demarcado predominantemente pelo conhecimento biológico.

A gente não, assim, e tem, tem que ter o conhecimento, né? Pelo menos, assim, das doenças que tem uma, as doenças sexualmente transmissível, a hipertensão, a diabetes, todas as doenças que você trabalha, tem que ter o conhecimento, sabe, saber alguma coisa, pra você poder orientar, né? E ter a técnica, saber como você vai preparar tal medicação, como cê vai fazer aquilo, aquele curativo, como, tudo cê tem que ter técnica. Tem que ter formação... (A06).

Também foram mencionados como recursos no trabalho os trabalhadores médicos e enfermeiras da UBS, o amparo da vigilância sanitária e a equipe do Serviço de Assistência Domiciliar da Secretaria Municipal de Saúde de Ribeirão Preto. As duas últimas indicações podem evidenciar que esses trabalhadores estão ampliando a rede de instrumentos que operam na atenção à saúde, ao citarem recursos externos à UBS. Esta ação é favorável à atenção à saúde na perspectiva da integralidade, pois amplia as ofertas para atender às necessidades da população.

No entanto, é preciso criar espaços para discutir e refletir sobre a inserção de outros instrumentos, pois a assistência social, os centros comunitários e as Organizações não Governamentais foram citados pelos AEs e TEs, porém não foram reconhecidos como tecnologias do processo de trabalho na ABS. $\mathrm{Na}$ produção do cuidado, acionar esses recursos não pode ficar na dependência dos trabalhadores lembrarem ocasionalmente, é necessário incorporá-los ao processo de trabalho no cotidiano dos serviços de saúde.

As tecnologias leves foram referidas como recursos do trabalho na ABS. A conversa foi tomada como um meio utilizado para conhecer melhor o usuário, reconhecer suas particularidades, individualidades e necessidades.

...às vezes a pessoa chega a gente tem que, às vezes numa conversa que a gente tem com a pessoa a gente consegue descobrir muita coisa que a pessoa tá necessitando, tá buscando no posto, pra gente poder produzir, passá aquilo que a pessoa tá passando mesmo (A04).

Considerar o escutar, ouvir, falar e conversar como recursos da ABS indica potencialidade para produzir cuidado na perspectiva da integralidade da atenção, pois para produzir cuidado integral é necessário articular o conjunto das tecnologias duras, leve-duras e leves, sendo que as tecnologias leves operam em todos os momentos da assistência.

Para alguns trabalhadores AEs e TEs ouvir, falar, conversar e escutar não são ferramentas legítimas do processo de trabalho em saúde, sendo estas adjetivadas como algo popular, fora do arcabouço próprio de um profissional da saúde.

O único recurso que a gente tem é a conversa, né? A papoterapia, e o médico atrás da gente com medicação. Só isso (A02).

É necessário valorizar o uso dessas tecnologias como recurso do trabalho na saúde por meio da qualificação da escuta e da oferta de estratégias e recursos adequados para assistir a população pela qual a UBS é responsável. Com isso, veremos o aumento da resolubilidade das ações, o incentivo à autonomia dos trabalhadores e o estímulo à criatividade, o que pode consolidar a atenção na perspectiva da integralidade, ao permitir que o trabalhador passe a se apropriar do produto de seu trabalho, tornando-se também responsável por esta produção.

Consideramos que o uso das tecnologias leves tem se apresentado como uma estratégia diante do esgotamento dos recursos disponíveis na UBS que focam o doente e a doença como a consulta médica, os medicamentos, as orientações de dietas, os encaminhamentos e também diante do sentimento de impotência no ato de cuidar frente à complexidade das necessidades trazidas pelos usuários.

\section{O produto do trabalho em saúde}

Os trabalhadores AEs e TEs apontaram como produto do trabalho na $\mathrm{ABS}$ o bem-estar dos usuários, entendido como 
evitar adoecimentos, evidenciando uma concepção de saúde como ausência de doenças.

Eu produzo assim, o bem-estar do paciente, um bom relacionamento, né? A prestação de serviço, né? De acordo que eu tenho a disponibilidade, né? Eu acho que é isso, proporciono assim, a saúde e o bem-estar deles, né? Colocando que a prevenção é, melhor você preveni, né? Antes, do que remediá, ficar doente, a importância da prevenção, do cuidado, né? Eu vejo que seria isso... (A01).

Também foram reconhecidos como produtos os procedimentos técnicos, o que converge com o objeto de trabalho focado na queixa do usuário.

Tirando esta parte de medicação que a gente faz, né? Tirando a parte de vacina, assim, o serviço técnico de fazer medicação e tal... (A03).

A tomada do bem-estar como ausência de doenças e dos procedimentos técnicos como produtos do trabalho na $\mathrm{ABS}$ são coerentes com a identificação das necessidades dos usuários como enfrentar doenças ou problemas de saúde. Essas percepções remetem novamente à fragmentação do homem que necessita de cuidados ora para alguma parte do corpo doente, ora para a mente que também se encontra doente ou com problemas. Revela, também, a fragmentação do processo de trabalho, condição desfavorável ao desenvolvimento da atenção à saúde na perspectiva da integralidade.

O conforto mental dos usuários também foi relatado como produto do trabalho na ABS, e esta produção é marcada como uma característica da enfermagem, ou seja, os trabalhadores AEs e TEs se reconhecem como agentes capazes de produzir o conforto mental do usuário.

Eu acredito que a gente seja, como o próprio nome diz, auxiliar, a gente também auxilia o médico no conforto físico do paciente, às vezes, até mental, porque, quando, muitos pacientes chegam aqui pra falar com o auxiliar, ele não tem um problema físico, ele qué conversar, ele qué chora, ele qué desabafa, aí é aonde a gente entra, pra promover conforto, né (A02)?

Observamos, novamente, o uso e a valorização das tecnologias leves sendo que, agora, estas tecnologias apresentam resultados na produção em saúde e, assim, abrem espaços para a produção do cuidado, na perspectiva da integralidade da atenção.

\section{Finalidade do trabalho em saúde}

A finalidade do trabalho em saúde foi predominantemente referida pelos AEs e TEs como ligada à oferta de recursos que recuperem o corpo doente e previnam doenças para evitar gastos por parte dos governos. Mesmo a promoção do bem-estar é tomada como ausência de doenças.

Deparamo-nos, também, com a finalidade do trabalho na ABS como o controle da ocorrência de doenças para evitar a incapacidade para o trabalho e a recuperação da força de trabalho para colocá-la de volta no processo de trabalho.

Prá, prá diminuir o número de doenças, prá não dar tanto gasto para o Estado, porque se você promove saúde, você elimina gastos. É, prá que a pessoa tenha uma vida digna, né? Prá que ela tenha condições de produzir, de se divertir, de ficar com a família, de cuidar da própria vida. (...) Produzir trabalhando, né? Fora ou dentro de casa, ou cuidando de uma pessoa mais velha, ou cuidando de criança, mas promover, né (A02)?

Os trabalhadores AEs e TEs também identificaram como finalidade do trabalho na ABS proporcionar melhores condições de vida aos usuários, garantir a saúde como direito de todos e dever do Estado e assistir quem não pode pagar o sistema privado.

Ah, eu acho que é uma obrigação porque existindo essa necessidade os governantes têm a obrigação de promover isso pro cidadão porque da mesma forma que investe em educação, investe em informação, ele também tem que investir na saúde, porque é fundamental como é que uma pessoa que não está bem fisicamente ou psicologicamente ele pode produzir em alguma outra, é uma fase que ela não é produtiva em área nenhuma, nem na escola, nem no trabalho, nem em lugar nenhum, é fundamental que ela esteja se sentindo bem prá que ela seja uma boa mãe, um bom funcionário, um bom médico, ser um bom professor, um bom advogado, um bom lavrador, ela tem que tá com a saúde compensada, equilibrada prá ela poder produzir em outras áreas (A09).

Evidencia-se um descompasso entre os discursos e as ações dos trabalhadores AEs e TEs, pois o que foi verbalizado como finalidade, prevenir doenças e promover bem-estar, foi pouco identificado em ações pontuais, circunscritas a determinadas situações. Na prática desses trabalhadores, predominaram as ações voltadas para a recuperação do corpo doente.

Deixamos aqui registrado que a emancipação dos sujeitos e o desenvolvimento da cidadania são dimensões que ainda estão muito distantes da finalidade do trabalho em saúde. Portanto, o processo de trabalho dos AEs e TEs desta UBS também se encontra distante do projeto de saúde cuja finalidade tem como ideário a integralidade, como é o caso do SUS.

\section{CONSIDERAÇÕES FINAIS}

Este estudo buscou caracterizar o processo de trabalho dos AEs e TEs de uma UBS, analisar as concepções desses trabalhadores sobre os elementos constitutivos do processo de trabalho na $A B S$, na perspectiva da integralidade da atenção, e identificar e analisar os aspectos dificultadores e facilitadores para desenvolver o processo de trabalho, na perspectiva da integralidade.

No processo de trabalho desses agentes, foi possível identificar duas composições distintas, sendo que encontramos de 
modo predominante a organização do processo de trabalho para atender pessoas com necessidade de enfrentar doenças/ problemas de saúde, mas também encontramos potencialidades para o desenvolvimento do processo de trabalho na direção da integralidade da atenção.

Para que se continue avançando nessa direção, consideramos ser necessário implementar a educação permanente no cotidiano do trabalho na $\mathrm{ABS}$ e, dentro desta prática, qualificar a escuta dos trabalhadores que atuam nesses serviços.

Consideramos necessário, ainda, ampliar os fóruns de discussões e as ações, incluindo como agentes as universidades, as instituições formadoras de AEs e TEs, os órgãos representativos da enfermagem e as instâncias políticas nos níveis nacional, estadual e municipal, para promover transformações estruturais na formação dos trabalhadores de nível médio de enfermagem, de modo que estes desenvolvam as competências necessárias para o trabalho na ABS, na perspectiva da integralidade da atenção.

Acreditamos que a concepção de competência, articulada à sustentação teórica do processo de trabalho em saúde, pode ser um dos caminhos para ampliar a qualificação dos trabalhadores, em especial dos AEs e TEs que compõem parte significativa do conjunto dos trabalhadores que produzem a atenção nos serviços de saúde e estão diuturnamente na linha de frente dos serviços, construindo o SUS e, sem dúvida, contribuindo com muita energia no fortalecimento de seus princípios.

\section{REFERÊNCIAS}

1. Silva JPV, Lima JC. Sistema Único de Saúde: Reafirmando seus Princípios e Diretrizes para Fazer Avançar a Reforma Sanitária Brasileira. In: Escola Politécnica de Saúde Joaquim Venâncio, organizador. Textos de apoio em políticas de saúde. Rio de Janeiro: Editora Fiocruz; 2005. p. 43-75.

2. Pereira MJB. Avaliação das características organizacionais e de desempenho das unidades de atenção básica em saúde do Distrito Oeste do município de Ribeirão Preto [tese]. Ribeirão Preto (SP): Escola de Enfermagem de Ribeirão Preto, Universidade de São Paulo; 2008.

3. Ministério da Saúde (BR). Cadastro Nacional dos Estabelecimentos de Saúde no Brasil. [citado em 2011 Set 09]. Disponível em: tabnet.datasus.gov.br/cgi/deftohtm. exe?cnes/cnv/prid02br.def

4. Mishima SM, Pereira MJB, Fortuna CM, Matumoto S. Trabalhadores de Saúde: Problema ou possibilidade de reformulação do trabalho em saúde? Alguns aspectos do trabalho em saúde e da relação gestor/trabalhador. In: Falcão A, Santos Neto PM, Costa PS, Belisário SA, organizadores. Observatório de Recursos Humanos em Saúde no Brasil: estudos e análises. Rio de Janeiro: Brasil/Ministério da Saúde; 2003. p. 137-156.

5. Almeida MCP, Mello DF, Neves, LAS. O trabalho de enfermagem e sua articulação com o processo de trabalho em saúde coletiva - rede básica de saúde em Ribeirão Preto. Rev Bras Enferm. 1991; 44 (2/3): 64-75.

6. Pereira MJB, Fortuna CM, Mishima SM, Almeida MCP, Matumoto S. A enfermagem no Brasil no contexto da força de trabalho em saúde: perfil e legislação. Rev Bras Enferm. 2009; 62(5): 771-777.

7. Mendes-Gonçalves RB. Práticas de Saúde: Processo de Trabalho e Necessidades. In: Cadernos CEFOR, São Paulo: Secretaria Municipal de Saúde de São Paulo; 1992.

8. Instituto Brasileiro de Administração Municipal. Escola Nacional de Serviços Urbanos. Núcleo de Saúde. A saúde no município: organização e gestão. Rio de Janeiro: IBAM/UNICEF; 1991.

9. Minayo MCS. O desafio do conhecimento: pesquisa qualitativa em saúde. 8a ed. São Paulo: Hucitec; 2004.

10. Merhy EE. A perda da dimensão cuidadora na produção da saúde: uma discussão do modelo assistencial e da intervenção no seu modo de trabalhar a assistência. Campinas: DMPS/FCM/UNICAMP; 1997.

11. Ministério da Saúde (BR). Humaniza SUS: Acolhimento com avaliação e classificação de risco. Brasília: Ministério da Saúde; 2004.

12. Ministério da Saúde (BR). Política nacional de atenção às urgências. Brasília: Ministério da Saúde; 2006. 